\title{
Calculation of Coupled Vibroacoustics Response Estimates from a Library of Available Uncoupled Transfer Function Sets
}

\author{
Andrew Smith. ${ }^{1}$ \\ NASA/Marshall Space Flight Center, Huntsville, AL, 35612 \\ Bruce LaVerde ${ }^{2}$ \\ ERC, Huntsville, AL, 35612 \\ Ron Hunt ${ }^{3}$ \\ Triumph Aerospace, Huntsville, AL, 35612 \\ and \\ Clay Fulcher, ${ }^{4}$ Robert Towner, ${ }^{5}$ Emmett Mcdonald ${ }^{6}$ \\ Jacobs Engineering, Huntsville, AL, 35612
}

\begin{abstract}
This technical paper describes an analysis tool that utilizes a database of transfer functions (TFs) to generate quick turn-around vibroacoustic response estimates suitable for developing initial vibration environment vendor specifications during the early stages of a new launch vehicle design program. The tool draws TFs from a database, combines TFs, and multiplies these by input excitations to estimate vibration responses. Initially, the database is populated with two sets of uncoupled TFs, the first set representing the vehicle panel unloaded vibration response TF, designated as $\mathrm{H}^{\alpha}$, and the second set representing the freefree component equipment (component only, no vehicle panel) vibration response TF, designated as $\mathbf{H}^{\beta}$. For a particular configuration undergoing analysis, the appropriate $\mathbf{H}^{\alpha}$ and $H^{\beta}$, are selected and coupled to generate an integrated TF, designated as $\mathbf{H}^{\alpha+\beta}$. This integrated TF, $\mathrm{H}^{\alpha+\beta}$, is then used with the appropriate input excitations to estimate vibration responses. This simple, but powerful, tool enables a user to estimate vibration responses without directly using finite element models, as long as suitable $\mathbf{H}^{\alpha}$ and $\mathbf{H}^{\beta}$ sets are defined in the database libraries. The paper will discuss the preparation of databases and provide the assumptions and methodologies necessary to combine $\mathbf{H}^{\alpha}$ and $\mathbf{H}^{\beta}$ sets into an integrated $\mathrm{H}^{\alpha+\beta}$. Validation of the coupled TFs will also be presented.
\end{abstract}

\section{Nomenclature}

$\mathrm{APTF}=$ Acceleration $/$ Pressure Transfer Function

$\mathrm{DAF}=$ Diffuse Acoustic Field

eq $=$ equation

$\mathrm{FEM}=$ Finite Element Model

MAC $=$ Modal Assurance Criteria

MSFC $=$ Marshall Space Flight Center

\footnotetext{
${ }^{1}$ Vibroacoustics Specialist, MSFC/EV31, Marshall Space Flight Center, AIAA Member

${ }^{2}$ Vibroacoustics Lead Engineer, Support to MSFC/EV31, Address/Mail Stop, and AIAA Member.

${ }^{3}$ Structural Dynamics \& Loads Specialist, Triumph Aerospace, 1500 Perimeter Parkway, Huntsville, AL, AIAA Member.

${ }^{4}$ Structural Dynamics \& Loads Specialist, Jacobs Engineering, 1500 Perimeter Parkway, Huntsville, AL, AIAA Member.

${ }^{5}$ Structural Dynamics \& Loads Specialist, Jacobs Engineering, 1500 Perimeter Parkway, Huntsville, AL, AIAA Member.

${ }^{6}$ Structural Dynamics \& Loads Specialist, Jacobs Engineering, 1500 Perimeter Parkway, Huntsville, AL, AIAA Member.
} 
NESC $=$ NASA Engineering and Safety Center

$\mathrm{RMM}=$ Response Matching Method

$\mathrm{SPL}=$ Sound Pressure Level

SPCD $=$ Secondary Component Loads Development Program

\section{Transfer Function Designations:}

$\mathrm{H}^{\alpha}$, - Exterior Vehicle Panel Unloaded Response Transfer Function defined at Interface Response Locations

$\mathrm{H}^{\beta}$ - Free-Free Component Equipment Response Transfer Function from Interface Base Excitations

$\mathrm{H}^{\alpha+\beta}$ - Integrated System Response Transfer Function from excitation over surface of Exterior Vehicle Panel

$H_{a_{j} / p_{b}}(\omega)$ is the transfer function between acceleration at point $j$ and pressure $p_{b}$ on patch $b$,

$\zeta_{m}$ is the critical damping ratio for mode $m$,

$F_{k}$ is the static force at point $k$ associated within a unit pressure on patch $b$,

Modal Data:

$\Phi_{j}=\left[\begin{array}{llll}\phi_{j 1} & \phi_{j 2} & \cdots & \phi_{j M}\end{array}\right]$ - Mode shapes for modes $1-M_{\text {for bare skin at a few reference locations }} j$,

$\omega=\left[\begin{array}{llll}\omega_{1} & \omega_{2} & \cdots & \omega_{M}\end{array}\right]$ - Natural frequencies for modes $1-M$ of bare skin,

$\tilde{\Phi}_{\ell}=\left[\begin{array}{llll}\tilde{\phi}_{\ell 1} & \tilde{\phi}_{\ell 2} & \cdots & \tilde{\phi}_{\ell \tilde{M}}\end{array}\right]$ - Mode shapes for modes $1-\tilde{M}$ for component-loaded skin at locations $\ell$,

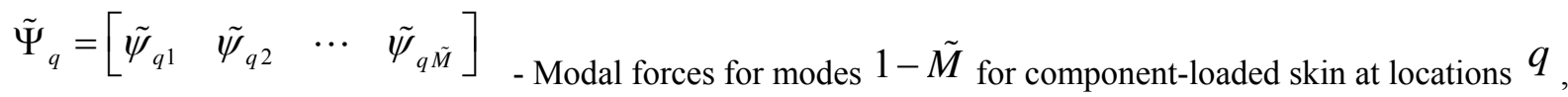

$\tilde{\omega}=\left[\begin{array}{llll}\tilde{\omega}_{1} & \tilde{\omega}_{2} & \cdots & \tilde{\omega}_{\tilde{M}}\end{array}\right]$ - Natural frequencies for modes $1-\tilde{M}$ of component-loaded skin.

$\phi_{j m}$ is the $m^{\text {th }}$ mass-normalized mode shape at response point $j$,

$\phi_{k m}$ is the $m^{\text {th }}$ mass-normalized mode shape at point $k$ in the pressure patch,

$\omega$ is the circular frequency,

$\omega_{m}$ is the circular natural frequency of mode $m$,

$M$ is the number of retained modes.

Static Data:

$F_{b}=\left[\begin{array}{llll}F_{b 1} & F_{b 2} & \cdots & F_{b N_{b}}\end{array}\right]$,

$b=1,2, \ldots N_{p} \quad$ - Force distribution on GRIDs for unit pressure on patches $b$,

$R_{b c}=\sqrt{\left(x_{b}-x_{c}\right)^{2}+\left(y_{b}-y_{c}\right)^{2}+\left(z_{b}-z_{c}\right)^{2}}$ - Distance between CGs of patches $b$ and $c$.

Pressure Data:

$P_{\text {ref }}(\omega)$ - Reference pressure autospectrum.

$N_{b}$ is the number of GRIDs in the pressure patch, 
$W_{b b}(\omega)$ - Scaling functions for non-uniform pressure autospectra over the entire skin. Calculate these to coincide with the zone autospectra specified in the Vibroacoustic Loads Databook, if available. That is, use the same $W_{b b}$ on patches within specified launch vehicle zones. Set $W_{b b}$ to unity if unknown.

\section{Introduction}

DROVIDING suitable vibration requirements for launch vehicle equipment is a challenge faced by each new Program. NASA's Engineering and Safety Center (NESC) has identified methodology for producing vibration response estimates for mass loaded vehicle panels as one of the top risks for new vehicle programs, observing that several different methodologies are in use that may generate differing response predictions. Program managers need realistic estimates for vibroacoustic response environments so that vibration environment specifications can be provided for the design of hardware. Accurate prediction of vibration environments in early stages of a new program helps to assure adequacy of the hardware to withstand the expected environments without specifying overly-severe environments. Specifying environments that are overly-conservative can drive up costs and add weight to the vehicle design. Providing realistic vibration environments can reduce schedule impacts, as the hardware does not require extreme design measures to withstand the vibration environments and environmental tests may be easier to perform. Imposing less severe test requirements may expand the number of test facilities that are able to satisfy required test levels. Engineering organizations engaged in a new vehicle design need a process that is quick to implement and easy to use. A complicated approach may present a schedule that it would be wiser to avoid. Therefore, engineering would like a proven process that provides seamless cross-cutting capability. The aim of this paper is to make such a methodology more accessible, as a capability, to the entire vibroacoustic loads community. Providing a tool that produces reliable vibration response estimates and is easy to use is the motivation for this work.

\section{Background}

In response to the NESC's identification of a critical technological need, the authors have presented validated methodologies for calculating both vibration response and dynamic loads for equipment mounted to vehicle exterior panels. ${ }^{9}$ The validation was based on acoustic response tests of a flight-like vehicle panel configured with different equipment masses. ${ }^{10,11,12} \mathrm{We}$ are seeking to make that demonstrated methodology more accessible as a capability to the wider loads community.

\section{Innovations for the End User}

The motivation for development of this tool is to address both the need and the vision to provide "vibroacoustic response estimates," using the most accurate game-changing estimation techniques, while minimizing complexities for the end-user. While a more straight-forward, but limiting, approach could be utilized that uses just a single transfer function representing a specific case, the goal of the proposed versatile approach is to provide a multitransfer function capability that enables an end-user to address and investigate several trade configurations with varying complexities.

The proposed approach is intended to be simple for the end-user, no longer requiring the end-user to work with finite element models. This capability is accomplished by leveraging off the work of others that have previously prepared the models and entered the response characteristics of these models into the database library. Since the unloaded panel and the component equipment transfer functions are generated separately, the end-user has numerous integrated mass-loaded panel options available. Integrated transfer functions are generated automatically in the tool by coupling the unloaded panel and component equipment panel transfer functions using the numerical procedure outlined herein. The end-user makes the following menu selections:

\footnotetext{
${ }^{9}$ Peck, J., p 5-21.

${ }^{10}$ Smith, A.,.

${ }^{11}$ Driskill, T..,.

12 Rodgers, C.,.
} 
1. The appropriate $\mathrm{H}^{\alpha}$ set - which depends on:

a. Zonal Exterior Vehicle Panel

b. Number and location of Interfacing connections to Single Equipment Assembly

2. The appropriate $\mathrm{H}^{\beta}$ set

a. Number and location of Interfacing connections of Single Equipment Assembly to primary vehicle panel

b. Equipment Assembly Mass and CG.

c. Estimated Damping Spectrum

3. The appropriate SPL description of the Excitation Environment.

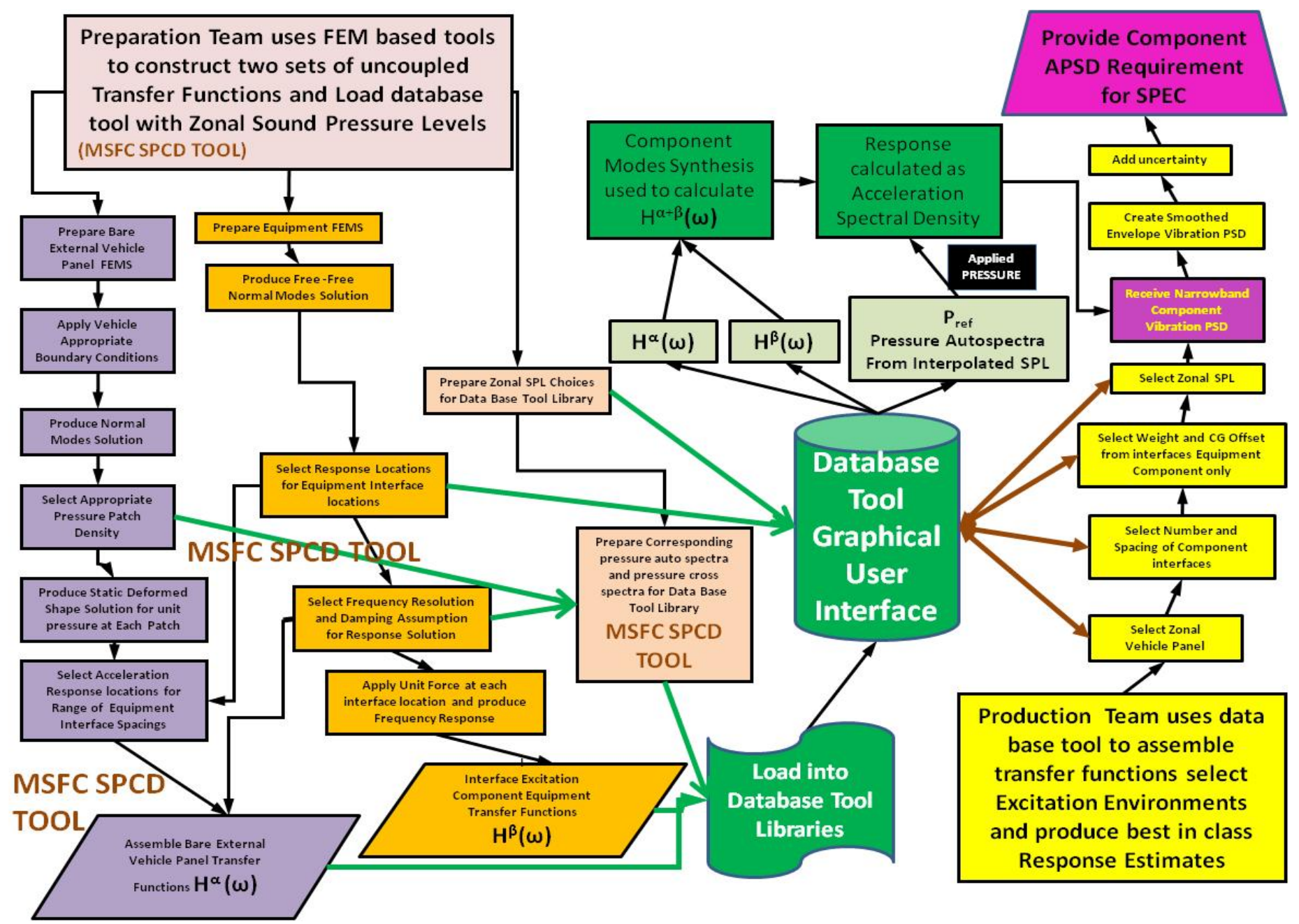

Figure 1. Process flow for Database Tool Preparation Team and Vibration Environment Preparation Team

From these simple inputs, the Database Tool constructs the $\mathrm{H}^{\alpha+\beta}$ TFs for the integrated system at each interface location. Next, narrowband vibration PSDs are calculated using the appropriate Pressure Auto- and Cross-Spectral densities that correspond to a selected zonal SPL at the interface nodes and provides them as a narrow band spectrum.

In order to provide the Max Expected Environment, the end-user may only need to construct a smoothed envelope of the narrow band PSDs output from the analysis tool, adding an appropriate uncertainty factor to result in vibration requirements suitable for a vendor specification. 
The calculated environment is intended to provide greater accuracy because more comprehensive design details are represented in the calculations than are typically considered using first-order estimates developed using traditional empirical scaling and mass attenuation techniques.

Figure 1 presents the expected process flow diagram to develop the tool, recommending two teams work in parallel. Efficient use of engineering resources may be achieved by assigning a small group to populate the Database Tool, "The Preparation Team," while assigning others to work on the tool itself, incorporating formulations, generating a GUI, and generating enveloping routines for requirement specification, "The Production Team." Since detailed component equipment interface details are not available until long after vendor specifications are released for a new vehicle program, development of a generic set of $\mathrm{H}^{\beta}$ is advised. These can be developed long before the actual component installation design details are decided. The generic transfer function set should include options that are realistic regarding the number and spacing of component equipment interfaces with the primary vehicle structure exterior panels. Preparing $\mathrm{H}^{\beta}$ for a wide range of weights and $\mathrm{CG}$ offsets is recommended. Once

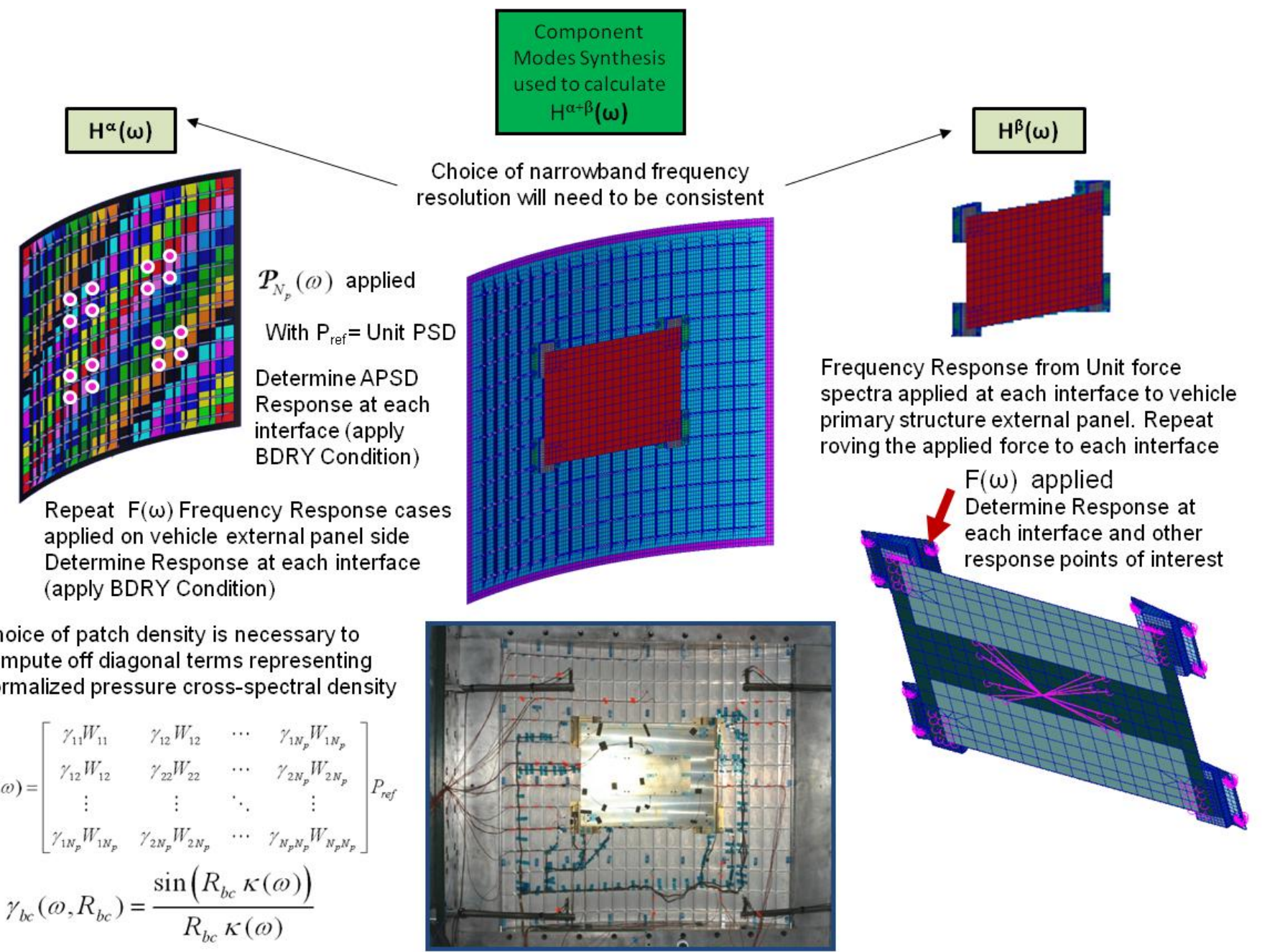

Figure 2. Notes on Setting up Uncoupled Transfer Function Ca

the primary structure design begins to solidify, a set of $\mathrm{H}^{\alpha} \mathrm{TF}$ would be prepared using the Acceleration/Pressure Transfer Function (APTF) method with a unit $\mathrm{P}_{\text {ref }}$ for the first Vehicle Zone (see equations 10 and 11). Guidance on calculating uncoupled TF is presented in Figure 2.

Equipped with generic $\mathrm{H}^{\beta}$ and vehicle design specific $\mathrm{H}^{\alpha}$ sets, the Production Team would begin to work in parallel to the preparation team. They would be able to provide both:

1. The Zonal Vibration Environments necessary as inputs to develop the Vehicle Program-Loads and Environments Data Book 
2. The vibration requirements necessary to issue vendor specifications for component design.

Figure 3 provides guidelines for boundary conditions to use in calculating the vehicle panel side transfer functions. The vibration environment estimates should be more accurate than those developed using the heritage approach, especially in important frequency ranges. A reduction in the weight and complexity of components and their attachment brackets may be possible by producing vibration environment estimates with greater accuracy.

\section{Basis Methodologies}

A pair of methodologies was previously presented by the Authors. ${ }^{13}$ The first method is the APTF, which applies a correlated pressure field across the surface of a vehicle panel. In this application, a Diffuse Acoustic Field (DAF) pressure assumption is adopted. Pressure spectra provided as "RMS sound pressure levels" are first converted to "pressure autospectral density". Then cross-spectra associated with the pressure field excitation are calculated according to the best fit of for a DAF using Equation (4). To implement this method, the excited vehicle panel surface must be subdivided into pressure patches. The distance between the center of each patch is selected based on the wave number required to calculate cross-spectra phasing between each patch.

An acceleration response transfer function is first described in terms of an excitation on a single patch in Equation (5). But the transfer function is fully developed to include the spatial correlation of the pressure field across the many patches of the vehicle panel in eq (10).

The second method, the Response Matching Method (RMM), may be applied when the vibration response of a bare vehicle panel is a known input to the problem. Several exterior vehicle panel response transfer functions, each defined at appropriate interface response locations, $\mathrm{H}^{\alpha}$, can be obtained using a portion of eq (10). Using the finite element method it is also possible to calculate a corresponding transfer function, $\mathrm{H}^{\alpha+\beta}$, for the integrated system (the same panel with equipment integrated on it) from eq (14).

One of the efforts involved in development of a working database tool will be to demonstrate the process of calculating the $\mathrm{H}^{\alpha+\beta}$, without using an integrated FEM of the exterior vehicle panel and the supported equipment. Instead, the $\mathrm{H}^{\alpha+\beta}$ is produced using the analysis tool, employing component mode synthesis from the union of selected $\mathrm{H}^{\alpha}$ and $\mathrm{H}^{\beta}$ options available within the library database.

The equations for the validated and very effective APTF and RMM approaches may be understood from the following equations and discussion. A random pressure field on a launch vehicle skin surface may be approximated by dividing the surface into "patches," or regions of uniform pressure with no phase offsets within the patch. The size of each patch must be chosen to be small enough to justify the assumption of uniform (though dynamically varying) pressure with zero phase offset across the patch. The selection of the appropriate patch and element size is not included in this monograph, but is addressed in a separate study.

The pressure may be defined as a stationary Gaussian random field with spatially varying autospectral density. The pressures on any pair of patches may be correlated, exhibiting a non-zero cross-spectral density between them.

The random pressure field is thus a square Hermitian matrix of spectral densities of dimension $N_{p}$, the total number of pressure patches. The pressure autospectra occur on the diagonal of the matrix. The cross-spectra includes the offdiagonal terms. The random pressure field on all patches may be written as

$$
\mathcal{P}_{N_{p}}(\omega)=\left[\begin{array}{cccc}
P_{11} & P_{12} & \cdots & P_{1 N_{p}} \\
P_{21} & P_{22} & \cdots & P_{2 N_{p}} \\
\vdots & \vdots & \ddots & \vdots \\
P_{N_{p} 1} & P_{N_{p} 2} & \cdots & P_{N_{p} N_{p}}
\end{array}\right]
$$

\footnotetext{
${ }^{13}$ Peck, J., p 16-21.
} 
where $P_{b c}=P_{c b}^{*}$, and the asterisk denotes the conjugate operator. If spatial functions $\gamma(\omega, R)$ are defined that relate the autospectra to the cross-spectra, eq (1) may be written as

$$
\boldsymbol{P}_{N_{p}}(\omega)=\left[\begin{array}{cccc}
\gamma_{11} \hat{P}_{11} & \gamma_{12} \hat{P}_{12} & \cdots & \gamma_{1 N_{p}} \hat{P}_{1 N_{p}} \\
\gamma_{21} \hat{P}_{12} & \gamma_{22} \hat{P}_{22} & \cdots & \gamma_{2 N_{p}} \hat{P}_{2 N_{p}} \\
\vdots & \vdots & \ddots & \vdots \\
\gamma_{N_{p} 1} \hat{P}_{1 N_{p}} & \gamma_{N_{p} 2} \hat{P}_{2 N_{p}} & \cdots & \gamma_{N_{p} N_{p}} \hat{P}_{N_{p} N_{p}}
\end{array}\right]
$$

where $\hat{P}_{b c}=\sqrt{P_{b b} P_{c c}}$ and $\gamma_{b b}$ have been added to the diagonals for generalization. The expression for $\hat{P}_{b c}$ arises from an inequality requirement on the coherence, which states that

$$
0 \leq \frac{\left|P_{b c}(\omega)\right|^{2}}{P_{b b}(\omega) P_{c c}(\omega)} \leq 1.0
$$

For a diffuse field, the spatial functions $\gamma$ may be expressed as

$$
\gamma_{b c}\left(\omega, R_{b c}\right)=\frac{\sin \left(R_{b c} \kappa(\omega)\right)}{R_{b c} \kappa(\omega)}
$$

where $R_{b c}$ is the distance between the area CGs of patches $b$ and $c, \kappa(\omega)=\omega / C_{o}$, and $C_{o}$ is the speed of sound through the fluid medium adjacent to the patch material. The patch CGs are constrained to lie on the curved skin surfaces. When $b=c$, the spatial functions coincide with the patch autospectra, the distance $R$ between patches vanishes and $\gamma_{b b} \rightarrow 1.0$ in the limit as $R_{b b} \rightarrow 0$ (L'Hopital's Rule). Finally, the patch autospectra may be expressed as products of frequency-dependent scaling functions $W_{b b}(\omega)$ and an arbitrary reference autospectrum. (e.g., one of the patch autospectra could be selected, but this is not required.)

$$
P_{b b}(\omega)=W_{b b}(\omega) P_{r e f}(\omega)
$$

Substituting eq (5) into eq (2) results in an expression for the diffuse-field pressure model used for the lift-off environments (Cases $01-08)$ in this study:

$$
\boldsymbol{P}_{N_{p}}(\omega)=\left[\begin{array}{cccc}
\gamma_{11} W_{11} & \gamma_{12} W_{12} & \cdots & \gamma_{1 N_{p}} W_{1 N_{p}} \\
\gamma_{12} W_{12} & \gamma_{22} W_{22} & \cdots & \gamma_{2 N_{p}} W_{2 N_{p}} \\
\vdots & \vdots & \ddots & \vdots \\
\gamma_{1 N_{p}} W_{1 N_{p}} & \gamma_{2 N_{p}} W_{2 N_{p}} & \cdots & \gamma_{N_{p} N_{p}} W_{N_{p} N_{p}}
\end{array}\right] P_{r e f}
$$


where $W_{b c}(\omega)=\sqrt{W_{b b}(\omega) W_{c c}(\omega)}$. It is important to recognize that all of the pressure auto and cross-spectra represented in eqs (1) - (6) are known, and are related to the reference spectrum $P_{r e f}$ through the scaling functions. If the scaling functions are unknown, then the skin structure under consideration must be limited to regions with similar pressure autospectra and $W_{b b}$ must be set to unity. The scaling functions may also be grouped into fewer distinct sets for large regions showing uniform pressure autospectra. That is, many of the functions may be assigned the same value for various zones of the launch vehicle even if the zone is subdivided into many patches.

Now consider the acceleration frequency response $a_{u}(\omega)$ at a point $j$ on the skin to a unit oscillating pressure $p_{u}(\omega)=\cos (\omega t)$ on patch $b$. The frequency response is also the transfer function between any acceleration response $a_{j}(\omega)$ at $j$ to arbitrary pressure $p_{b}(\omega)$ on patch $b$. Note that the response point locations are unrestricted; they may be located either within or external to the patch. In NASTRAN, the unit pressure is distributed as a set of non-uniform forces on the grid points comprising the shell elements within the patch. This non-uniform force distribution for a unit pressure may be obtained with an OLOAD request at the patch GRIDs in NASTRAN SOL 101 (linear static loads solution). The force distribution may then be swept through the frequency range of interest to obtain the frequency response. The acceleration/pressure transfer function for a single patch may be expressed explicitly as the sum of weighted acceleration/force transfer functions. The weighting factor is the static force $F_{k}$ at each input location $k$ on the patch due to a unit pressure (from an OLOAD request in SOL 101):

$$
H_{a_{j} / p_{b}}(\omega)=\sum_{k=1}^{N_{b}}\left\{F_{k} \cdot \sum_{m=1}^{M}\left[\frac{-\omega^{2} \phi_{j m} \phi_{k m}}{\omega_{m}^{2}-\omega^{2}+i 2 \zeta_{m} \omega_{m} \omega}\right]\right\}
$$

where

$H_{a_{j} / p_{b}}(\omega)$ is the transfer function between acceleration at point $j$ and pressure $p_{b}$ on patch $b$,

$F_{k}$ is the static force at point $k$ associated with a unit pressure on patch $b$,

$\phi_{j m}$ is the $m^{\text {th }}$ mass-normalized mode shape at response point $j$,

$\phi_{k m}$ is the $m^{\text {th }}$ mass-normalized mode shape at point $k$ in the pressure patch,

$\omega$ is the circular frequency,

$\omega_{m}$ is the circular natural frequency of mode $m$,

$\zeta_{m}$ is the critical damping ratio for mode $m$,

$N_{b}$ is the number of GRIDs in the pressure patch,

$M$ is the number of retained modes.

The acceleration PSD response $A_{j b}(\omega)$ to a random pressure $P_{b b}(\omega)$ on patch $b$ is the squared magnitude of the acceleration/pressure transfer function in (7) multiplied by the pressure PSD, or

$$
\begin{aligned}
A_{j b}(\omega) & =\left|H_{a_{j} / p_{b}}(\omega)\right|^{2} P_{b b}(\omega) \\
& =\left|H_{a_{j} / p_{b}}(\omega)\right|^{2} W_{b b}(\omega) P_{r e f}
\end{aligned}
$$


The total response at location $j$ includes the autospectra from the pressures on all of the patches and also from non-zero cross-spectra between any two patches, or

$$
\begin{aligned}
A_{j}(\omega) & =\sum_{b}^{N_{p}}\left|H_{a_{j} / p_{b}}\right|^{2} P_{b b}+\sum_{b}^{N_{p}} \sum_{c \neq b}^{N_{p}} H_{a_{j} / p_{b}} H_{a_{j} / p_{c}}^{*} P_{b c} \\
& =\sum_{b}^{N_{p}} \sum_{c}^{N_{p}} H_{a_{j} / p_{b}} H_{a_{j} / p_{c}}^{*} P_{b c}
\end{aligned}
$$

Expressing eq (9) in terms of the reference spectrum and the spatially dependent cross-spectra of eqs (4) and (6), we obtain

$$
A_{j}(\omega)=\sum_{b}^{N_{p}} \sum_{c}^{N_{p}} \gamma_{b c} W_{b c} H_{a_{j} / p_{b}} H_{a_{j} / p_{c}}^{*} P_{r e f}=\sum_{b}^{N_{p}} \sum_{c}^{N_{p}} \frac{\sin \left(\kappa R_{b c}\right)}{\kappa R_{b c}} \sqrt{W_{b b} W_{c c}} H_{a_{j} / p_{b}} H_{a_{j} / p_{c}}^{*} P_{r e f}
$$

Note that the spatial functions $\gamma$ reduce to unity for $b=c$, as mentioned previously. Eq (10) may be expressed in matrix form for computational efficiency in Matlab as

$$
A_{j}(\omega)=\left[\mathrm{H}_{a_{j}} \sqrt{\mathrm{W}} \Gamma \sqrt{\mathrm{W}} \mathrm{H}_{a_{j}}^{\dagger}\right] P_{r e f}
$$

Where ${ }^{\dagger}$ denotes the Hermitian conjugate and

$\mathrm{H}_{a_{j}}(\omega)=\left[\begin{array}{llll}H_{a_{j} / p_{1}} & H_{a_{j} / p_{2}} & \cdots & H_{a_{j} / p_{N_{p}}}\end{array}\right](\mathrm{a} / \mathrm{p}$ transfer functions from eq (7)),

$\mathrm{W}(\omega)=\left[\begin{array}{cccc}W_{11} & & & 0 \\ & W_{22} & & \\ & & \ddots & \\ & & & W_{N_{p} N_{p}}\end{array}\right]$ (pressure autospectra scaling functions from eq (5)), 
$\Gamma(\omega)=\left[\begin{array}{ccc}1 \frac{\sin \left(\kappa R_{12}\right)}{\kappa R_{12}} & \cdots & \frac{\sin \left(\kappa R_{1 N_{p}}\right)}{\kappa R_{1 N_{p}}} \\ 1 & \cdots & \frac{\sin \left(\kappa R_{2 N_{p}}\right)}{\kappa R_{2 N_{p}}} \\ \mathrm{SYM} & \ddots & \vdots \\ & & 1\end{array}\right]$ (spatial functions from eq (4)).

If the pressure autospectrum does not vary significantly over the region of interest, $W_{b b}(\omega)=W_{c c}(\omega) \cong 1.0$, and eq (11) simplifies to

$$
A_{j}(\omega)=\left[\mathrm{H}_{a_{j}} \Gamma \mathrm{H}_{a_{j}}^{\dagger}\right] P_{r e f}
$$

The term inside the bracket in eq (11) (or eq (12) for uniform pressure spectra) may be considered the squared transfer function between the total response at location $j$ and the entire diffuse pressure field:

$$
\left|H_{a_{j} / p}(\omega)\right|^{2}=\mathrm{H}_{a_{j}} \sqrt{\mathrm{W}} \Gamma \sqrt{\mathrm{W}} \mathrm{H}_{a_{j}}^{\dagger}=\frac{A_{j}(\omega)}{P_{r e f}(\omega)}
$$

Note that $H_{a_{j} / p}$ is not an unchanging characteristic of the system since the scaling functions $\mathrm{W}$ depend upon the generally non-uniform patch autospectra, which change at different times in the launch trajectory. However, if the random pressure may be considered uniform over the surface of interest, $H_{a_{j} / p}$ is characteristic of the system independent of input pressure or the output acceleration.

Eq (13) may be used to calculate $A_{j}(\omega)$ if the reference pressure $P_{\text {ref }}$ and scaling functions $\mathrm{W}$ are known. Conversely, an effective reference pressure $\bar{P}_{r e f}(\omega)$ may be calculated if the acceleration PSD is specified and W is set to the identity matrix. The locations $j$ selected as reference points on a skin without mounted components should be minimized, and chosen as far as possible from zone boundaries, joints, or local skin features such as cutouts, or doublers (which could distort the results).

Now consider the case of a component mounted directly to the outer skin of a launch vehicle. The applied pressure does not change, but the transfer function in eq (13) developed for the bare skin must be generated for the component-loaded skin. Eqs (7) - (13) are applied at a response location $\ell$ using the modes of the componentloaded skin, and eq (13) becomes

$$
\left|\tilde{H}_{a_{\ell} / p}(\omega)\right|^{2}=\tilde{\mathrm{H}}_{a_{\ell}} \sqrt{\mathrm{W}} \Gamma \sqrt{\mathrm{W}} \tilde{\mathrm{H}}_{a_{\ell}}^{\dagger}=\frac{\tilde{A}_{\ell}(\omega)}{P_{r e f}(\omega)}
$$


where the tilde denotes the component-loaded acceleration/pressure transfer function and skin response. If the pressure is unknown, but the acceleration on the unloaded skin is known from measured flight data, the componentloaded response at any point $\ell$ may be obtained by eliminating $P_{\text {ref }}(\omega)$ from eqs (13) and (14):

$$
\tilde{A}_{\ell}(\omega)=A_{j}(\omega) \cdot \frac{\left|\tilde{H}_{a_{\ell} / p}(\omega)\right|^{2}}{\left|H_{a_{j} / p}(\omega)\right|^{2}}
$$

where the acceleration/single-patch pressure transfer functions $H_{a_{j} / p_{b}}(\omega)$ in eq (13) for the bare skin are obtained from eq (7), and $\tilde{H}_{a_{\ell} / p_{b}}(\omega)$ in eq (14) for the component-loaded skin from

$$
\tilde{H}_{a_{\ell} / p_{b}}(\omega)=\sum_{k=1}^{N_{b}}\left\{F_{k} \cdot \sum_{m=1}^{\tilde{M}}\left[\frac{-\omega^{2} \tilde{\phi}_{\ell m} \tilde{\phi}_{k m}}{\tilde{\omega}_{m}^{2}-\omega^{2}+i 2 \tilde{\zeta}_{m} \tilde{\omega}_{m} \omega}\right]\right\}
$$

Equation (15) defines the RMM for predicting the response of a component-loaded skin structure when the bare skin response is known, and the acceleration/pressure transfer functions for both bare and component-loaded skins are available from modal analysis of the respective finite element models, or from modal testing. The frequencydependent ratio of transfer functions in (15) replaces the Barrett scaling factor given by

$$
B=\frac{w_{n}}{w_{n}+w_{c}}
$$

where $w_{n}$ and $w_{c}$ are the weights of the bare and component-loaded structures, respectively.

Force and moment responses at specified interface elements (e.g., CBUSH) may be obtained in the same fashion by replacing the first mode shape term in eq (16) with the modal forces and moments obtained in a NASTRAN RESTART in SOL 103. The $-\omega^{2}$ term in the numerator of eq (16) is also dropped. The expression for the response force (or moment) at location $q$ is similar to that for acceleration in (15):

$$
\tilde{F}_{q}(\omega)=A_{j}(\omega) \cdot \frac{\left|\tilde{H}_{f_{q} / p}(\omega)\right|^{2}}{\left|H_{a_{j} / p}(\omega)\right|^{2}}
$$

where $\tilde{F}_{q}(\omega)$ is the force (or moment) PSD at location $q$, and $\tilde{H}_{f_{q} / p}$ is the transfer function between the total force (or moment) at location $q$ and the pressure:

$$
\left|\tilde{H}_{f_{q} / p}(\omega)\right|^{2}=\tilde{\mathrm{H}}_{f_{q}} \sqrt{\mathrm{W}} \Gamma \sqrt{\mathrm{W}} \tilde{\mathrm{H}}_{f_{q}}^{\dagger}=\frac{\tilde{F}_{q}(\omega)}{P_{r e f}(\omega)}
$$

The individual transfer functions $\tilde{H}_{f_{q} / p_{b}}(\omega)$ in $\tilde{\mathrm{H}}_{f_{q}}$ between the force at location $q$ and each single-patch pressure on any patch $b$ are given by 


$$
\tilde{H}_{f_{q} / p_{b}}(\omega)=\sum_{k=1}^{N_{b}}\left\{F_{k} \cdot \sum_{m=1}^{\tilde{M}}\left[\frac{\tilde{\psi}_{q m} \tilde{\phi}_{k m}}{\tilde{\omega}_{m}^{2}-\omega^{2}+i 2 \tilde{\zeta}_{m} \tilde{\omega}_{m} \omega}\right]\right\}
$$

where $\tilde{\psi}_{q m}$ is the $m^{\text {th }}$ modal force at location $q$.

\section{Validation}

Validation of the APTF method was provided in a previous technical presentation ${ }^{14}$. Therefore the RMM is featured in the validation section of this work. Examples will be supplied that overlay predictions from the direct APTF calculation with predictions from the RMM calculation. Furthermore, results calculated using the combined uncoupled transfer functions will also be compared to results calculated with the full-up coupled system transfer functions. These demonstration examples are currently in work.

\section{Conclusion}

This paper presents an expansion upon successful validations of the APTF and RMM methods. The accomplishment provides a simple, but powerful, database analysis tool for the MSFC vibroacoustics team to estimate vibration responses at equipment mounting locations instead of using heritage processes that typically require running detailed analysis models and/or obtaining/processing substantial ground and flight test data. This puts cross-cutting capability into the hands of the propulsion and vehicle system departments to provide input vibration environment requirements for a new launch vehicle program.

\section{Acknowledgments}

The Authors would like thank and acknowledge Ms. Karen Oliver, and Mr. Phillip Harrison of MSFC/ EV31, as well as Mr. Greg Frady of MSFC EV41 without whose support and vision the work could not have been accomplished. Thanks are also necessary for the team that has developed the SPCD Tool which automates much of the work that the database tool preparation team would have to accomplish. These team members include Mr. Clay Fulcher, Mr. Ron Hunt, Mr. Douglas Jones, and Mr. Nick Mastramico.

\section{References}

${ }^{1}$ Peck, J., Smith, A., Fulcher, C.., LaVerde B., Hunt, R.., "Development of Component Interface Loads on a Cylindrical Orthogrid Vehicle Section from Test-Correlated Models of a Curved Panel," Proceedings of 2011 Spacecraft and Launch Vehicle Dynamic Environments Workshop, June 2011..

${ }^{2}$ Maasha, R., Towner, R., LaVerde B., Band, J., "Preliminary Correlation Results Summary for Mass Loaded Vehicle Panel Test Article Finite Element Models and Modal Survey Tests," Proceedings of 2011 Spacecraft and Launch Vehicle Dynamic Environments Workshop, June 2011.

${ }^{3}$ Majed, A., Henkel, E., Kolaini, A., “An Improved Method of Random Vibration Mass Attenuation," Presentation by Applied Structural Dynamics \& JPL to the NESC Face to Face Meeting, March 2011.

\footnotetext{
${ }^{14}$ Peck, J., p 5-13.
} 


\title{
Calculation of Coupled Vibroacoustics Response Estimates from a Library of Available Uncoupled Transfer Function Sets
}

\author{
Andrew M. Smith, ${ }^{*}$ R. Benjamin Davis, ${ }^{\dagger}$ \\ NASA Marshall Space Flight Center, Huntsville, AL, 35812, USA \\ Bruce T. LaVerde, \\ Ronald A. Hunt, $\S$ \\ ERC, Inc., Huntsville, AL, 35812, USA \\ Triumph Aerospace, Huntsville, AL, 35812, USA \\ Douglas C. Jones, " and Jonathan L. Band ॥ \\ Jacobs Engineering, Huntsville, AL, 35812, USA
}

The design and theoretical basis of a new database tool that quickly generates vibroacoustic response estimates using a library of transfer functions (TFs) is discussed. During the early stages of a launch vehicle development program, these response estimates can be used to provide vibration environment specification to hardware vendors. The tool accesses TFs from a database, combines the TFs, and multiplies these by input excitations to estimate vibration responses. The database is populated with two sets of uncoupled TFs; the first set representing vibration response of a bare panel, designated as $H^{s}$, and the second set representing the response of the free-free component equipment by itself, designated as $H^{c}$. For a particular configuration undergoing analysis, the appropriate $H^{s}$ and $H^{c}$ are selected and coupled to generate an integrated TF, designated as $H^{s+c}$. This integrated TF is then used with the appropriate input excitations to estimate vibration responses. This simple yet powerful tool enables a user to estimate vibration responses without directly using finite element models, so long as suitable $H^{s}$ and $H^{c}$ sets are defined in the database libraries. The paper discusses the preparation of the database tool and provides the assumptions and methodologies necessary to combine $H^{s}$ and $H^{c}$ sets into an integrated $H^{s+c}$. An experimental validation of the approach is also presented.

\section{Nomenclature}

Symbols

A Acceleration power spectral density

a Acceleration response

$f \quad$ External force

$H \quad$ Transfer function

$k \quad$ acoustic wavenumber

$M \quad$ Number of retained modes

$N_{p} \quad$ Number of pressure patches

$P \quad$ Pressure spectral density

$\hat{P} \quad$ Pressure spectral density magnitude

$P_{\text {ref }} \quad$ Pressure reference spectrum

\footnotetext{
*Aerospace Engineer, Vehicle Loads and Strength Branch, Mail Code EV31, MSFC, AL

${ }^{\dagger}$ Aerospace Engineer, Structural and Dynamics Analysis Branch, Mail Code ER41, MSFC, AL

¥Aerospace Engineer, ERC, Inc., 4901 Corporate Dr NW E Huntsville, AL, AIAA Member

$\S$ Aerospace Engineer, Triumph Aerospace Systems, P.O. Box 9025, MSFC, AL 35812

『Aerospace Engineer, Jacobs Engineering, 1500 Perimeter Parkway, Huntsville, AL

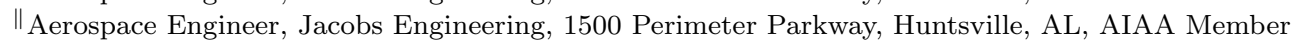




\begin{tabular}{|c|c|}
\hline$p$ & Pressure \\
\hline$r$ & Distance from field point to response point \\
\hline$W$ & Pressure scaling functions \\
\hline$\alpha$ & Areas associated with the degrees of freedom in a patch \\
\hline$\Gamma$ & Matrix of pressure field spatial function \\
\hline$\gamma$ & Pressure field spatial function \\
\hline$\zeta$ & Linear viscous damping ratio \\
\hline$\phi$ & Eigenvector \\
\hline$\omega$ & Circular frequency \\
\hline \multicolumn{2}{|c|}{ Superscript } \\
\hline$c$ & Component \\
\hline$s$ & Structure \\
\hline \multicolumn{2}{|c|}{ Subscript } \\
\hline$b$ & Interface points \\
\hline$e$ & Excitation points \\
\hline$j, k$ & Pressure patch indices \\
\hline$l$ & Degree of freedom index associated with single pressure patch \\
\hline$m$ & Mode number index \\
\hline$N_{p}$ & Total number of pressure patches \\
\hline$p$ & Non-interface points \\
\hline$\varepsilon$ & Pressure patches \\
\hline \multicolumn{2}{|c|}{ Conventions } \\
\hline()$^{*}$ & Complex conjugate \\
\hline[]$^{\dagger}$ & Complex conjugate transpose \\
\hline \multicolumn{2}{|c|}{ Acronyms } \\
\hline DAF & Diffuse acoustic field \\
\hline LFP & Large footprint \\
\hline $\mathrm{MFP}$ & Medium footprint \\
\hline MSFC & Marshall Space Flight Center \\
\hline NESC & NASA Engineering and Safety Center \\
\hline RMM & Response Matching Method \\
\hline RMS & Root mean squared \\
\hline RPTF & Response to Pressure Transfer Function Method \\
\hline PSD & Power Spectral Density \\
\hline SFP & Small footprint \\
\hline $\mathrm{TF}$ & Transfer function \\
\hline
\end{tabular}

\section{Introduction}

PROviding suitable vibration requirements for launch vehicle equipment is a challenge faced by each new 1 program. NASA's Engineering and Safety Center (NESC) has identified methodology for producing vibration response estimates for mass-loaded vehicle panels as one of the top risks for new vehicle programs, observing that several different methodologies are currently in use with the potential to generate differing response predictions. Program managers need realistic estimates for vibroacoustic response environments so that vibration environment specifications can be developed for the design of hardware. Accurate prediction of vibration environments in early stages of a new program helps to assure adequacy of the hardware to withstand the expected environments without specifying overly severe environments. Specifying environments that are overly conservative can increase financial and schedule costs as well as add weight to the vehicle design. Further, imposing less severe environments may result in hardware tests that can be performed more readily and with reduced cost.

Generating accurate vibroacoustic predictions has historically required detailed analysis models and/or obtaining and processing substantial ground and flight test data. All of these activities require far more time and resources than are typically available at the start of a launch vehicle development program. Consequently, engineering organizations engaged in a new vehicle design need a vibration environment estimation process 
that is quickly implemented and easily used. This paper describes a new tool that is being developed to fill this need. The methodology described here can quickly produce reliable vibration response estimates without requiring detailed analysis or test data.

\section{Background and Motivation}

Different organizations in the vibroacoustics community apply a variety of methods to estimate vibration environments for vehicle exterior panels. These environments are induced by fluctuating pressure fields on the panel from launch acoustics or flow-induced turbulent boundary layers during ascent. A review of the various methods in 2009, with concurrence by the NESC, indicated that when a panel is not loaded with mounted equipment, the different approaches returned results that were similar and consistent 1 Conversely, when vibration environments were considered for exterior panels with equipment attached, commonly referred to as mass-loaded panel systems, the predicted environments varied widely with little consistency across these same organizations. Such wide dissimilarity in the predicted environments throughout the community creates a high degree of uncertainty, and the NESC carried this finding forward as a risk for new launch vehicle (LV) development $1 / 2$ The risk has been addressed by the authors and others ${ }^{3 / 6}$

In response to the NESC's identification of a critical technological need, the authors have presented a pair of validated methodologies for calculating both vibration response and dynamic loads for equipment mounted to vehicle exterior panels 4 4 7 The validation was based on acoustic response tests of a flight-like vehicle panel configured with different equipment masses. The first methodology is known as the Response to Pressure Transfer Function (RPTF) method. RPTF applies a correlated pressure field across the surface of a vehicle panel. In this application, a diffuse acoustic field (DAF) pressure assumption is adopted. Pressure spectra provided as RMS sound pressure levels are first converted to pressure autospectral density. Then cross-spectra associated with the pressure field excitation are calculated according to the best fit for a DAF. To implement this method, the excited vehicle panel surface must be subdivided into pressure patches. The distance between the center of each patch is selected based on the wave number required to calculate crossspectra phasing between each patch. An acceleration response transfer function is first described in terms of an excitation on a single patch, but the transfer function is fully developed to include the spatial correlation of the pressure field across the many patches of the vehicle panel.

The second method, the Response Matching Method (RMM), provides the basis for much of the theoretical development presented here. The key difference between RMM and the present methodology is that previous versions of RMM have only been able to accept fully coupled TFs. The methodology presented in Section IV] calculates the same response in terms of the uncoupled TFs of the bare vehicle panel and the attached component alone. This formulation enables panels and components to be combined in any desired configuration without the need for TFs specific to each configuration.

RPTF and RMM have been developed such that they each accept as input one of the two most common forms of data available in the historical archive for past and present LVs:

1. Zonal sound pressure levels are provided as standard requirements during the development phase of a new vehicle design and can be used as inputs to the RPTF method.

2. Acceleration measurements on large-acreage exterior panels are often collected during the development flight tests of a new vehicle. Autospectral densities are often available either directly from a test flight or as an adjusted estimate from a similar vehicle design. These may be applied as inputs for predicting mass-loaded panel responses using RMM.

Software that implements RPTF and RMM already exists, but requires that the analyst has access to the complete coupled form of the system equations for the mass-loaded panel of interest. The theoretical development in Section [V] shows how RPTF and RMM can be extended to solve the coupled system equations while using the uncoupled system equations as inputs.

Table 1 compares RPTF and RMM in their two forms. Each method has a form that accepts coupled TFs and a form that accepts uncoupled TFs. RPTF and RMM in both their forms offer the ability to accurately predict random responses; not only acceleration but also velocity, displacement, panel and component stress and strain, interface force, or any other linear output expressed as a set of modal eigenvectors. The new development of allowing RPTF and RMM to accept uncoupled TFs allows the user to quickly and accurately evaluate vibration environments for a wide variety of component configurations and interface designs without the need for costly and time-consuming updates to a large system FE model. 
Table 1. Methods for populating the launch vehicle database tool library.

\begin{tabular}{cllll}
\hline Method & $\begin{array}{l}\text { Transfer } \\
\text { Functions }\end{array}$ & $\begin{array}{l}\text { Modal } \\
\text { Requirements }\end{array}$ & Type of Input & Available Output \\
\hline \multirow{2}{*}{ RPTF } & Coupled & Loaded Structure & $\begin{array}{l}\text { Pressure Auto Spectral } \\
\text { Density }\end{array}$ & $\begin{array}{l}\text { Any Response Auto and } \\
\text { Cross Spectral Densities }\end{array}$ \\
\hline \multirow{2}{*}{ RMM } & Coupled & $\begin{array}{l}\text { Unloaded } \\
\text { Structure and } \\
\text { Loaded Structure }\end{array}$ & $\begin{array}{l}\text { Unloaded Skin } \\
\text { Acceleration Auto } \\
\text { Spectral Density }\end{array}$ & $\begin{array}{l}\text { Any Response Auto } \\
\text { Spectral Density }\end{array}$ \\
\hline \multirow{2}{*}{ RPTF } & \multirow{2}{*}{ Uncoupled } & $\begin{array}{l}\text { Unloaded } \\
\text { Structure and } \\
\text { Component }\end{array}$ & $\begin{array}{l}\text { Pressure Auto Spectral } \\
\text { Density }\end{array}$ & $\begin{array}{l}\text { Any Response Auto and } \\
\text { Cross Spectral Densities }\end{array}$ \\
\hline \multirow{2}{*}{ RMM } & \multirow{2}{*}{ Uncoupled } & $\begin{array}{l}\text { Unloaded } \\
\text { Structure and } \\
\text { Component }\end{array}$ & $\begin{array}{l}\text { Unloaded Skin } \\
\text { Acceleration Auto } \\
\text { Spectral Density }\end{array}$ & $\begin{array}{l}\text { Any Response Auto } \\
\text { Spectral Density }\end{array}$ \\
\hline
\end{tabular}

\section{Description of Database Tool}

Extending RPTF and RMM to accept uncoupled TFs as inputs enables the development of a new database tool to predict the mass-loaded panel response for a new LV. The database tool allows an engineer who may not be well-versed in finite element methods to quickly visualize the narrow band vibration response of a given primary structure panel with mounted components selected from a library. Each component is integrated into the vehicle panel automatically, with no requirement to create a new FE model. The library of available components that can be integrated into the vehicle panel will span a realistic range of weight, centroud offset, and interface footprint.

A typical example of a component library for a specific LV zone is shown in Table 2. The size of the footprint for each component is designated as small (SFP), medium (MFP) or large (LFP). When a component is selected, the database tool generates the coupling equations derived from the unloaded panel and component natural frequencies and modes and then applies the panel pressure or acceleration input to predict the mass-loaded panel response. If more detailed modal data for the component is known from either test or FE analysis, this information may be used instead of the pre-loaded library components.

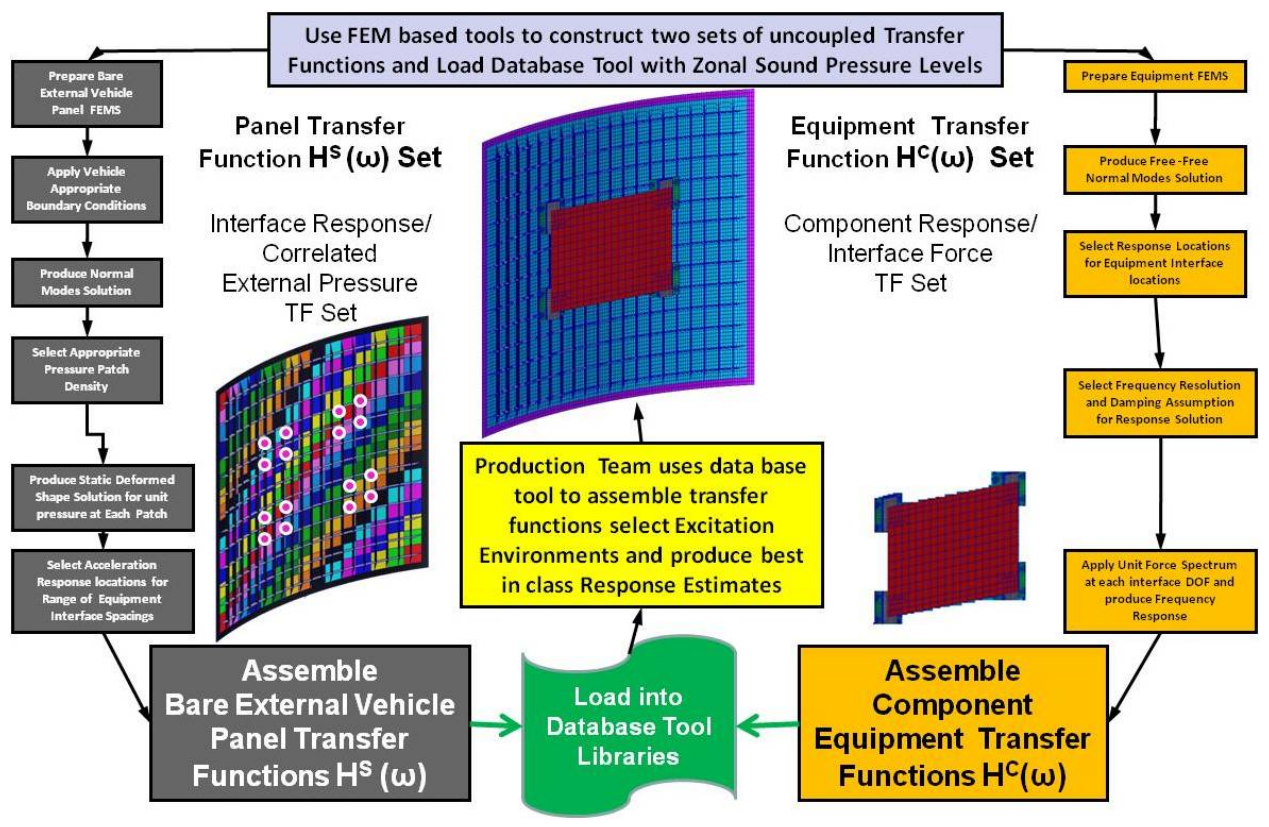

Figure 1. Process flow for preparing the database tool 
Table 2. Example of possible choices for components in the database tool library

\begin{tabular}{|c|c|c|c|c|}
\hline $\begin{array}{c}\text { Component } \\
\text { Designation }\end{array}$ & $\begin{array}{c}\text { Number of } \\
\text { Interfaces }\end{array}$ & $\begin{array}{c}\text { Interface } \\
\text { DOF }\end{array}$ & $\begin{array}{c}\text { Weight } \\
\text { (lb) }\end{array}$ & $\begin{array}{c}\text { Centroid } \\
\text { Offset (in) }\end{array}$ \\
\hline 1-SFP-15-8 & 4 & 24 & 15.00 & 8.00 \\
\hline 2-SFP-30-8 & 4 & 24 & 30.00 & 8.00 \\
\hline 3-SFP-45-8 & 4 & 24 & 45.00 & 8.00 \\
\hline 4-SFP-15-11 & 4 & 24 & 15.00 & 11.00 \\
\hline 5-SFP-30-11 & 4 & 24 & 30.00 & 11.00 \\
\hline 6-MFP-30-8 & 8 & 48 & 30.00 & 8.00 \\
\hline 7-MFP-45-8 & 8 & 48 & 45.00 & 8.00 \\
\hline 8-MFP-60-8 & 8 & 48 & 60.00 & 8.00 \\
\hline 9-MFP-15-11 & 8 & 48 & 15.00 & 11.00 \\
\hline 10-MFP-45-11 & 8 & 48 & 45.00 & 11.00 \\
\hline 11-LFP-45-11 & 16 & 96 & 45.00 & 11.00 \\
\hline 12-LFP-60-11 & 16 & 96 & 60.00 & 11.00 \\
\hline
\end{tabular}

Figure 1 shows the process flow for preparing the database tool library. The curved panel in this illustration depicts the 16 interface locations for a LFP component. The tool generates the coupled system response from uncoupled panel and component transfer functions derived from the pre-computed or measured modes. The database tool preparation team generates the necessary and consistent data for the library from finite element models or measurements and defines default values for several user-accessible database tool parameters.

The database tool is designed to anticipate the need to expand in scope and complexity as time passes and a LV program matures. In the beginning of a LV development program the primary structure designs may be known in rigorous detail. The component design and integration details may not be as well-defined. A set of default component options should be sufficient to quickly develop reasonable vibration environments, qualification test specifications, and preliminary cost estimates early in the program. As more detailed information becomes available, higher-fidelity FE models of the actual component and integration details are easily installed in the database for more precise and accurate estimates of the vibration environments.

The database tool end-user does not create a model or perform an FE analysis. Instead, he or she makes reasonable selections from panel structures and components loaded in the library. He or she also selects the flight event of interest (e.g., lift-off, ascent). The selected flight event and the vehicle zone containing the selected panel will correspond to two sets of source environments from which the user may choose one: either the sound pressure level or unloaded panel acceleration spectral density.

After making these selections and accepting the default setting for a damping schedule the database tool will calculate the coupled system transfer functions from the uncoupled primary structure and component modal characteristics. The theoretical basis for the coupling methodology is provided in the next section.

The benefit of assembling the loaded panel transfer functions from the separate unloaded panel and component transfer functions is the ability to predict the loaded panel vibration environment without requiring an update of the full system modal solution. Thus, the computationally expensive modal solution of the vehicle is required only once for a vehicle design cycle, and components may be selected from the default database tool library or provided anew as the component design matures. But lunch is never free. To achieve reasonable accuracy in the final response prediction from decoupled transfer functions, more modes need to be retained for both the unloaded structure and the component models. (Preliminary data suggest that

$$
5 \text { of } 14
$$


the mode retention requirements are especially large for the component models.) Further refinement of the database tool will incorporate well-known component mode synthesis techniques to alleviate some of the added computational burden.

\section{Methodology}

We begin by deriving an expression for the response of a structural panel with an attached component. The relation is written in terms of the individual transfer functions associated with the panel and component. Since the database tool contains only individual panel and component transfer functions, writing the combined transfer function in this manner is essential. Similar derivations have been presented by Majed, et al ${ }^{6}$ Next, the pressure field acting on the structure is considered. By conveniently expressing the pressure field in terms of known quantities, the acceleration spectral density of the component loaded panel can be found. It is this expression, Eq. 20, that underpins the core computations of the database tool.

\section{IV.A. Response of Structural Panel with an Attached Component}

Consider a structural panel with an attached component as shown in figure 2. The panel is subject to force excitation at $e$ locations and has interface points with the attached component at $b$ locations. A frequency dependent transfer function, $H(\omega)$, is defined simply as the ratio of the acceleration response to the input (e.g., $H \equiv a / F)$. In this case, the input is an external force; pressure inputs will be considered subsequently. Given the definition of the transfer function, the response of a structure is generally given by

$$
\mathbf{a}=[H]\{\mathbf{f}\},
$$

where the explicit frequency dependance has been dropped for convenience. Partitioning Eq. 1 to consider the response at $b$ interface locations and $p$ non-interface points gives

$$
\mathbf{a}=\left\{\begin{array}{c}
\mathbf{a}_{\mathbf{b}} \\
\cdots \\
\mathbf{a}_{\mathbf{p}}
\end{array}\right\}=\left[\begin{array}{ccc}
H_{b e}^{s} & \vdots & H_{b b}^{s} \\
\cdots & \cdots & \cdots \\
H_{p e}^{s} & \vdots & H_{p b}^{s}
\end{array}\right]\left\{\begin{array}{c}
\mathbf{f}_{e} \\
\cdots \\
\mathbf{f}_{\mathbf{b}}
\end{array}\right\}
$$

From a free-body diagram of the component, the forces at the interface are related to the accelerations by

$$
\mathbf{f}_{\mathbf{b}}=-\left[H_{b b}^{c}\right]^{-1} \mathbf{a}_{\mathbf{b}}
$$

Combining Eqs. (2) and (3) and solving for $\mathbf{a}_{\mathbf{b}}$ we may express the acceleration at the interface in terms of the interface transfer functions and the external force

$$
\mathbf{a}_{\mathbf{b}}=\left[\left[H_{b b}^{s}\right]^{-1}+\left[H_{b b}^{c}\right]^{-1}\right]^{-1}\left[H_{b b}^{s}\right]^{-1}\left[H_{b e}^{s}\right] \mathbf{f}_{\mathbf{e}}
$$

Now consider points $p$ that are located on the panel, but not at interface points. The acceleration response of such points is given by

$$
\mathbf{a}_{\mathbf{p}}=\left[H_{p e}^{s}\right] \mathbf{f}_{\mathbf{e}}+\left[H_{p b}^{s}\right] \mathbf{f}_{\mathbf{b}} .
$$

Using Eqs. (3) and (5) as well as acceleration continuity, the response at points $p$ is

$$
\mathbf{a}_{\mathbf{p}}=\left[\left[H_{p e}^{s}\right]-\left[H_{p b}^{s}\right]\left[\left[H_{b b}^{s}\right]+\left[H_{b b}^{c}\right]\right]^{-1}\left[H_{b e}^{s}\right]\right] \mathbf{f}_{\mathbf{e}}
$$

Eqs. (4) and (6) will be used in Section IV.C to find the response of the component loaded panel subject to random pressure excitation.

Now consider the response at a single point $p$ due to a single force excitation $e$. For linear structural systems, the complex scalar elements of the force/acceleration TF matrices shown in Eqs. (4) and (6) can be expressed as a summation of natural frequencies and modes

$$
H_{p e}=\sum_{m=1}^{M} \frac{-\omega^{2} \phi_{p m} \phi_{e m}}{\left(\omega_{m}^{2}-\omega^{2}+2 i \varsigma_{m} \omega_{m} \omega\right)},
$$




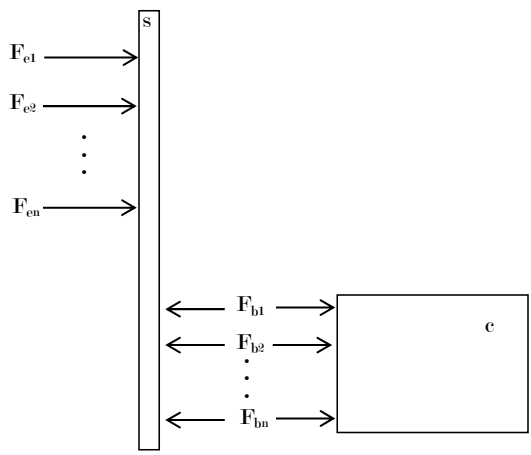

Figure 2. Schematic of structural panel (s) with attached component (c)

where the TF elements given by Eq. (7) take the same form for responses at interface and non-interface points. This form also applies regardless of whether one is assembling matrices of bare structure TFs or component TFs (provided, of course, that the appropriate structure or component natural frequencies and modes are used).

\section{IV.B. Pressure Excitation of Panel}

A random pressure field on a launch vehicle skin surface may be approximated by dividing the surface into patches, or regions of uniform pressure with no phase offsets within the patch. The size of each patch must be chosen to be small enough to justify the assumption of uniform (though dynamically varying) pressure with zero phase offset across the patch. The selection of the appropriate patch and element size is not included in this monograph, but is addressed in a separate study $[5]$

The pressure may be defined as a stationary Gaussian random field with spatially varying autospectral density. The pressures on any pair of patches may be correlated, exhibiting a non-zero cross-spectral density between them. The random pressure field is thus a Hermitian matrix of spectral densities of dimension $N_{p}$, the total number of pressure patches. The pressure autospectra occur on the diagonal of the matrix. The cross-spectra appear off of the diagonal. The random pressure field on all patches may be written as

$$
P(\omega)=\left[\begin{array}{cccc}
P_{11} & P_{12} & \cdots & P_{1 N_{p}} \\
P_{21} & P_{22} & \cdots & P_{2 N_{p}} \\
\vdots & \vdots & \ddots & \vdots \\
P_{N_{p} 1} & P_{N_{p} 2} & \cdots & P_{N_{p} N_{p}}
\end{array}\right]
$$

where $P_{j k}=P_{k j}^{*}$. If spatial functions $\gamma(\omega, r)$ are defined to relate the autospectra to the cross-spectra, Eq. (8) may be written as

$$
P(\omega)=\left[\begin{array}{cccc}
\gamma_{11} \hat{P}_{11} & \gamma_{12} \hat{P}_{12} & \cdots & \gamma_{1 N_{p}} \hat{P}_{1 N_{p}} \\
\gamma_{21} \hat{P}_{12} & \gamma_{22} \hat{P}_{22} & \cdots & \gamma_{2 N_{P}} \hat{P}_{2 N_{p}} \\
\vdots & \vdots & \ddots & \vdots \\
\gamma_{N_{p} 1} \hat{P}_{1 N_{p}} & \gamma_{N_{p} 2} \hat{P}_{2 N_{p}} & \cdots & \gamma_{N_{p} N_{p}} \hat{P}_{N_{p} N_{p}}
\end{array}\right]
$$

where $\hat{P}_{j k}=\sqrt{P_{j j} P_{k k}}$. As will be discussed shortly, in the case of a DAF, $\gamma_{j k}\left(\omega, r_{j k}\right)=1$ along the diagonals; however, the $\gamma$ terms have been included in the diagonal terms in Eq. 9 ) for generalization. The expression for $\hat{P}_{j k}$ satisfies an inequality requirement on the coherence which states that

$$
0 \leq \frac{\left|P_{j k}(\omega)\right|^{2}}{P_{j j}(\omega) P_{k k}(\omega)} \leq 1.0
$$


For a DAF, the spatial functions may be expressed as

$$
\gamma_{j k}\left(\omega, r_{j k}\right)=\frac{\sin \left(k r_{j k}\right)}{k r_{j k}}
$$

where the patch centroids are constrained to lie on the curved skin surfaces. When $j=k$ the spatial functions coincide with the patch autospectra and the gamma approaches unity by L'Hopital's Rule. Finally, the components of the pressure matrix may be expressed as products of frequency-dependent scaling functions, $W_{j k}$ and an arbitrary reference autospectrum pressure, $P_{r e f}$, i.e.,

$$
P_{j k}(\omega)=\gamma_{j k} W_{j k}(\omega) P_{r e f}(\omega)
$$

Substituting Eq. (12) into Eq. (9) we obtain an expression for the diffuse-field pressure model used for the lift-off environments in this study

$$
P(\omega)=\left[\begin{array}{cccc}
W_{11} & \gamma_{12} W_{12} & \cdots & \gamma_{1 N_{p}} W_{1 N_{p}} \\
\gamma_{12} W_{12} & W_{22} & \cdots & \gamma_{2 N_{p}} W_{2 N_{p}} \\
\vdots & \vdots & \ddots & \vdots \\
\gamma_{1 N_{p}} W_{1 N_{p}} & \gamma_{2 N_{p}} W_{2 N_{p}} & \cdots & W_{N_{p} N_{p}}
\end{array}\right] P_{r e f}
$$

or more compactly,

$$
P(\omega)=[W]^{\frac{1}{2}}[\Gamma][W]^{\frac{1}{2}} P_{r e f},
$$

where for a DAF,

$$
\Gamma(\omega)=\left[\begin{array}{cccc}
1 & \frac{\sin \left(k r_{12}\right)}{k r_{12}} & \cdots & \frac{\sin \left(k r_{1 N_{p}}\right)}{k r_{1 N_{p}}} \\
1 & \cdots & \frac{\sin \left(k r_{2 N_{p}}\right)}{k r_{2 N_{p}}} \\
& \mathrm{SYM} & \ddots & \vdots \\
& & & 1
\end{array}\right] .
$$

It is important to recognize that all of the pressure auto and cross-spectra discussed are related to the known reference spectrum, $P_{\text {ref }}$ through the scaling functions. If the acoustic field is not diffuse and the scaling functions are unknown, then the skin structure under consideration must be limited to regions with similar pressure autospectra and must be set to unity. The scaling functions may also be grouped into fewer distinct sets for large regions showing uniform pressure autospectra. That is, many of the functions may be assigned the same value for various zones of the launch vehicle even if the zone is subdivided into many patches.

\section{IV.C. Mass Loaded Panel System Response Due to Pressure Field Excitation}

The acceleration response of a panel at non-interface points $p$ due to a vector of patch pressures, $p_{\varepsilon}$ is

$$
\mathbf{a}_{\mathbf{p}}=\left[H_{p \varepsilon}^{s+c}\right]\left\{\mathbf{p}_{\varepsilon}\right\} .
$$

where $H_{p \varepsilon}^{s+c}$ denotes the acceleration/pressure TF of the component loaded panel. From Eq. (6), it follows that $H_{p \varepsilon}^{s+c}$ can be written in terms of acceleration/pressure TFs for the bare structure and component as

$$
\left[H_{p \varepsilon}^{s+c}\right]=\left[H_{p \varepsilon}^{s}\right]-\left[H_{p b}^{s}\right]\left[\left[H_{b b}^{s}\right]+\left[H_{b b}^{c}\right]\right]^{-1}\left[H_{b \varepsilon}^{s}\right] .
$$

Acceleration/pressure TFs can be related to acceleration/force TFs by considering the pressure on a patch as an array of distributed point forces. To then convert an acceleration/force TF to an acceleration/pressure TF it is only necessary to multiply by the surface area associated with each of the point forces on a patch. (Computing the surface area associated with a given node is a calculation that can be readily performed with a finite element software.) Using this TF conversion and Eq. (7), it is possible to write the individual elements of $\left[H_{p \varepsilon}\right]$ corresponding to response point $p$ and patch $\varepsilon$

$$
H_{p \varepsilon}=\sum_{m=1}^{M} \phi_{p m} \frac{-\omega^{2}\left\{\phi_{l m}\right\}^{T}\left\{\alpha_{l}\right\}}{\left(\omega_{m}^{2}-\omega^{2}+2 i \varsigma_{m} \omega_{m} \omega\right)},
$$


where $\left\{\phi_{l m}\right\}$ is a partial eigenvector containing the elements corresponding to the degrees of freedom associated with a single patch $\varepsilon$ and $\left\{\alpha_{l}\right\}$ is the vector of areas associated with those degrees of freedom.

Turning attention to the acceleration PSD response of a panel, the total response at a single point $p$ includes the autospectra from the pressures on all of the patches and also from non-zero cross-spectra between any two patches, $\frac{8}{8}$ or

$$
A_{p}=\sum_{j}^{N_{p}} \sum_{k}^{N_{p}} H_{p \varepsilon_{j}}^{*} H_{p \varepsilon_{k}} P_{j k} .
$$

Using Eqs. 14 and (19), the PSD response of a component loaded panel at point $p$ can by expressed in matrix-vector form for computational efficiency as

$$
A_{p}^{s+c}=\left\{H_{p \varepsilon}^{s+c}\right\}[W]^{\frac{1}{2}}[\Gamma][W]^{\frac{1}{2}}\left\{H_{p \varepsilon}^{s+c}\right\}^{\dagger} P_{r e f},
$$

where $\left\{H_{p \varepsilon}^{s+c}\right\}$ is a $1 \mathrm{x} N_{p}$ vector and $N_{p}$ is the number of pressure patches. The individual elements of $\left\{H_{p \varepsilon}^{s+c}\right\}$ are given by Eq. 18. Similarly, the PSD response of the bare structure is

$$
A_{p}^{s}=\left\{H_{p \varepsilon}^{s}\right\}[W]^{\frac{1}{2}}[\Gamma][W]^{\frac{1}{2}}\left\{H_{p \varepsilon}^{s}\right\}^{\dagger} P_{r e f} .
$$

If the pressure field applied to a component loaded structure is unknown, but the acceleration PSD response of the bare structure to the same pressure field is known, it is possible to combine Eqs. (20) and (21) to obtain a relation that is independent of pressure

$$
A_{p}^{s+c}=A_{p}^{s} \frac{\left\{H_{p \varepsilon}^{s+c}\right\}[W]^{\frac{1}{2}}[\Gamma][W]^{\frac{1}{2}}\left\{H_{p \varepsilon}^{s+c}\right\}^{\dagger}}{\left\{H_{p \varepsilon}^{s}\right\}[W]^{\frac{1}{2}}[\Gamma][W]^{\frac{1}{2}}\left\{H_{p \varepsilon}^{s}\right\}^{\dagger}} .
$$

\section{Equivalence of the RMM and the RPTF Method}

As discussed in Section III NASA's historical archive of source environments data for past and present launch vehicles contains data as either zonal sound pressure levels or acceleration autospectral densities corresponding to specific locations on the launch vehicle surface. The database tool will enable the choice of either of these inputs. When sound pressure levels are used, the mass-loaded panel environments will be calculated using RPTF. If the source environments are in the form of acceleration autospectral densities, then the output will be calculated using RMM. It is important to note that these are mathematically equivalent methodologies; their only substantive difference is the type of input that they accept. Figure 3 shows the equivalency of the RPTF method and RMM. Figure 3 (a) shows the source environment as a pressure power spectral density. This pressure environment was propagated through a bare panel using RPTF and the resulting acceleration power spectral density is shown in Figure 3 (b). Figure 3 (c) shows the acceleration power spectral density of the mass-loaded panel calculated using both methods. The RPTF solution (solid line) started with the pressure spectral density in Figure 3 (a) and used the uncoupled transfer function formulation of the method to calculate an acceleration power spectral density for the mass-loaded panel. The RMM solution (dashed line) started with the bare panel acceleration power spectral density curve shown in Figure 3 (b) and also used the uncoupled TF formulation to calculate a mass-loaded panel response curve. As expected, the two curves are identical. All of the presented source environments and responses correspond to the direction normal to the panel; however, the equivalency of the two methods in the other two axes can also be shown.

While the mathematical equivalency of the RMM and RPTF methods has been shown, it is important to note that in practice, the two methods often will not predict equivalent responses. This is due to the manner in which source environments are created and stored in the historical archives. Pressure spectral density source environments for lift-off acoustics are inherently smooth and broadband in nature. They are also relatively repeatable from launch to launch. Thus, when these environments are enveloped and stored in archives, only a modest amount of conservatism must be added to the spectrum to account for the uncertainty in the environment. This is not the case for acceleration power spectral density source environments. Due to the natural modes of the vehicle panels, these source environments tend to have many more discrete peaks. Furthermore, the frequency at which these discrete peaks occur will have some flightto-flight variability. In the process of enveloping these environments for storage in the archive, a relatively 


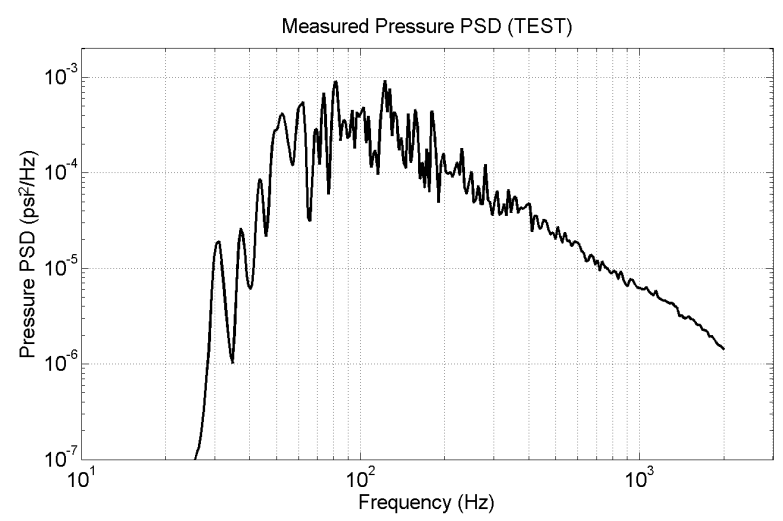

(a)

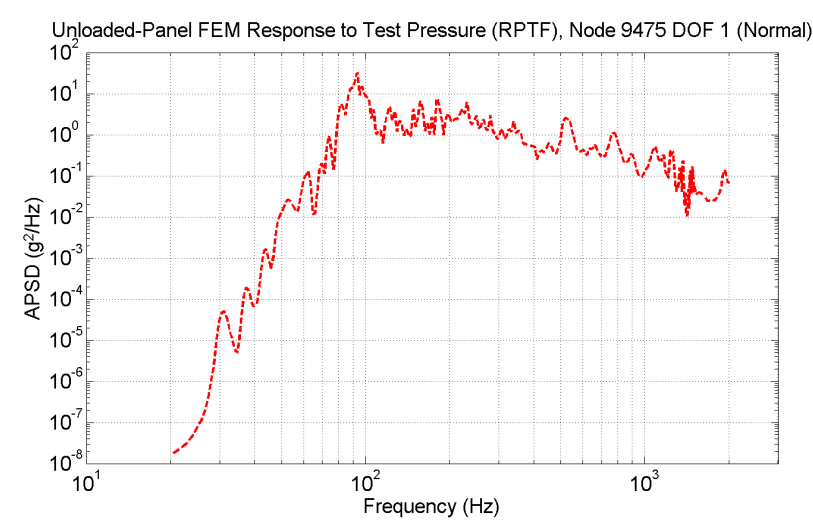

(b)

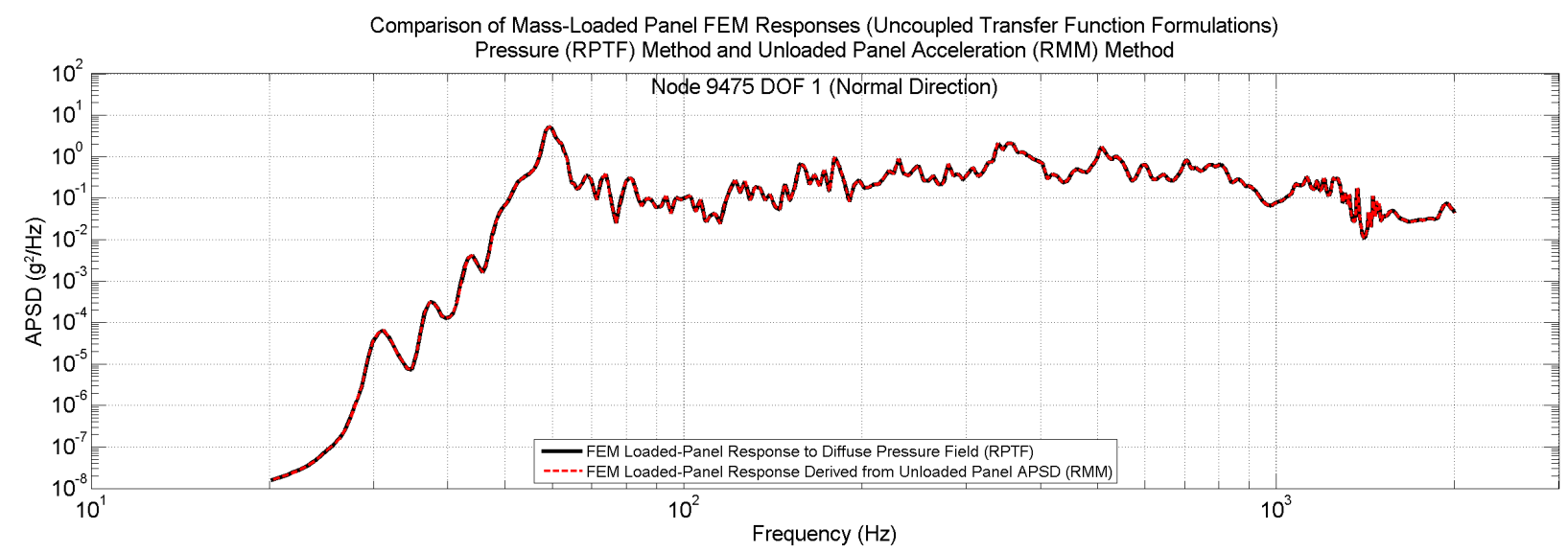

(c)

Figure 3. Equivalence of the RPTF method and RMM. (a) Pressure power spectral density source environment. (b) Equivalent source environment in terms of acceleration power spectral density. (c) Acceleration power spectral density of the mass-loaded panel calculated using both methods.

large amount of uncertainty is applied. Therefore, in practice, the use of RMM and acceleration power spectral density source environments will typically result in higher response spectra than will result from using RPTF and a corresponding pressure spectral density source environment. While both types of source environments may not always be available for a given launch vehicle panel of interest, if the analyst has a choice, it will generally be more conservative to use RMM with acceleration power spectral density source environments. This more conservative approach is typically warranted early in a launch vehicle development program. As a launch vehicle program matures, it may be possible to remove some conservatism from component requirement specifications by using RPTF with the appropriate pressure spectral density source environments.

\section{Implementation and Validation of Uncoupled TF Formulation}

To prove the viability of the database tool concept it is necessary to show that the response of a massloaded panel generated by the uncoupled TF formulation is approximately equivalent to the response generated by the coupled TF formulation. To show this approximate equivalence a finite element model of a flight-like panel with and without attached equipment assemblies was developed. A modal analysis was performed on these models using MSC/NASTRAN and the corresponding TFs (i.e., $H^{s}$ and $H^{s+c}$ ) were calculated using the appropriate equations (either Eq. (7) or Eq. 187. A free-free modal analysis of the attached equipment assembly model by itself was also performed to calculate the necessary component TFs (i.e., $H^{c}$ ). The finite element models were designed simulate a configuration recently ground tested in the acoustic chamber at MSFC. A companion paper by the authors details this test series and presents the 


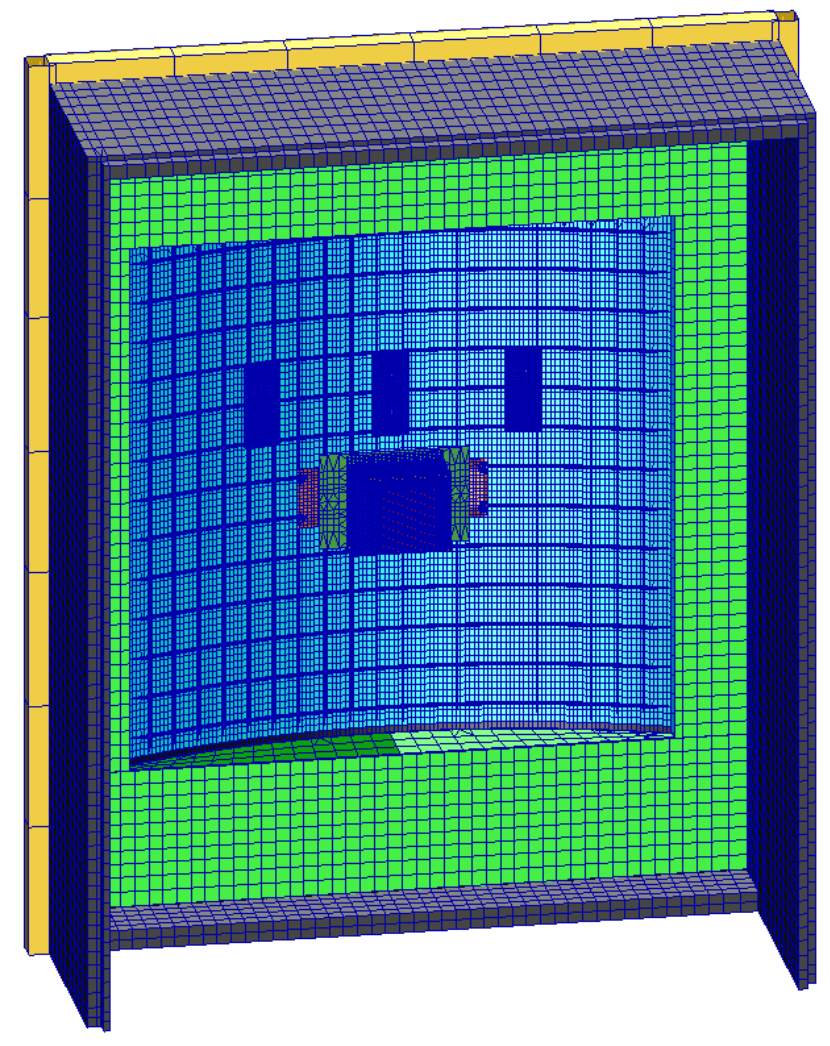

Figure 4. Oblique view of FE model of panel and surrounding support structure.

currently available data ${ }^{9}$ Figure 4 shows the finite element model of the panel and the attached equipment assemblies. The surrounding wall structure was also modeled and is shown here. This model contains 60,841 nodes and 57,313 elements. Checks were made to ensure the natural frequencies and mode shapes predicted by the models showed good agreement with those found in test. ${ }^{7}$

The test panel was subjected to acoustic noise excitation energies closely simulating the liftoff event and roughly approximating ascent flight events. This acoustic pressure spectrum was then used as an input to the RPTF method. The coupled TF and uncoupled TF formulations of the RPTF method were then used to calculate the response of the mass-loaded panel at an interface point. The results are overlaid in Figure 5. Note the excellent agreement between the responses across the entire frequency range. The agreement is not perfect, however. It is suspected that this discrepancy is due to the approximate nature of the uncoupled formulation used here. Other component mode synthesis approaches (e.g., the Craig-Brampton method ${ }^{10}$ ) would likely result in a more exact match. Implementing such approaches will be the subject of future work, but the agreement evidenced by figure 5 is more than adequate for the purpose of quickly developing vibroacoustic response estimates early in a vehicle program.

Responses were also calculated for a non-interface point on the panel. A point on the model was chosen to correspond to a location at which test data were available. The acceleration response spectra from the uncoupled RPTF formulation, the coupled RPTF formulation, and the test data are overlaid in figure 6 . The coupled and uncoupled formulations show excellent agreement with each other and the test data. This serves as further validation of the coupled RPTF formulation and the first ever validation of the uncoupled RPTF formulation. Since the uncoupled RMM formulation was shown in Section $\nabla$ to be mathematically equivalent to the uncoupled RPTF formulation, the validation of the uncoupled RPTF formulation implicitly validates the uncoupled RMM formulation as well. Note that the damping schedule used to calculate both analytical responses is a damping schedule identified from the test data using a software tool developed at 


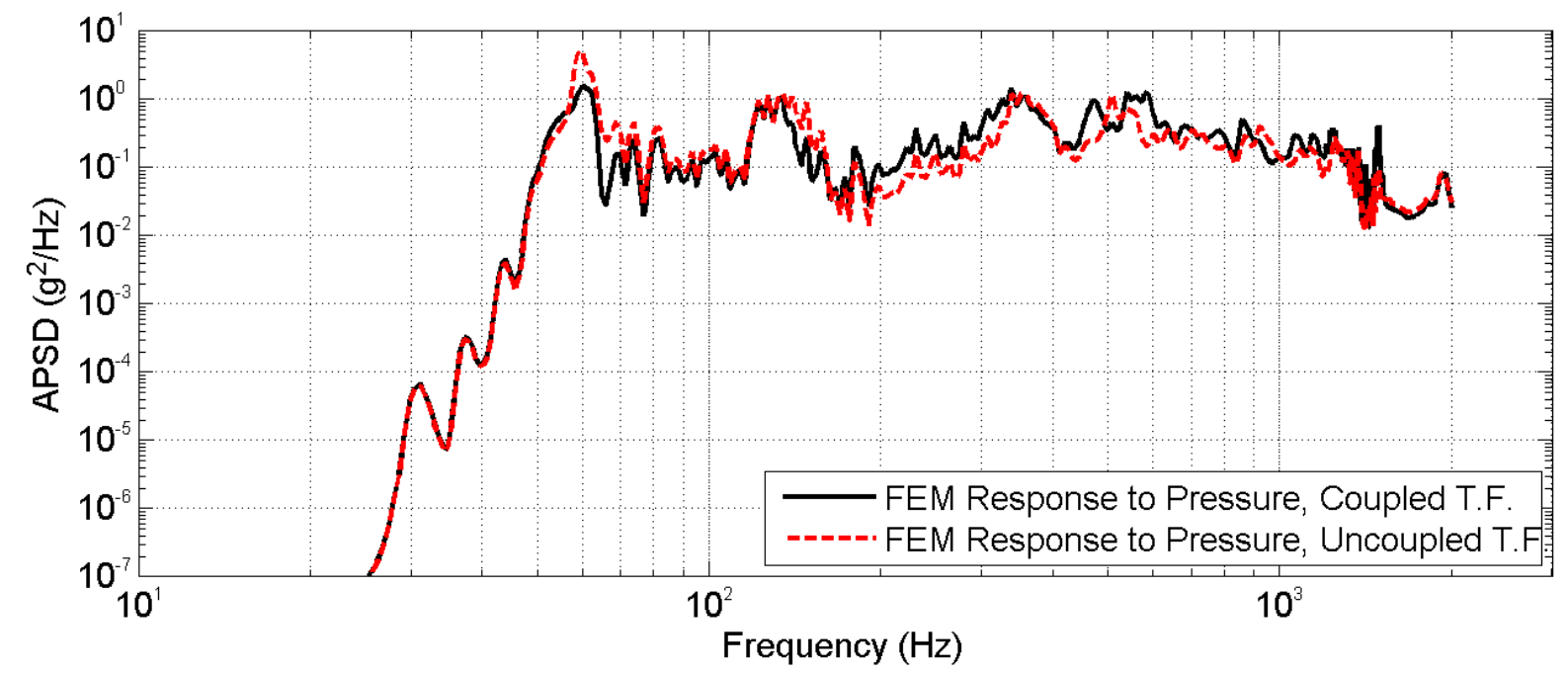

Figure 5. The response of a mass-loaded panel at an interface point calculated using the coupled TF and uncoupled TF formulations of the RPTF method.

MSFC known as DampID. More information concerning DampID is presented in Smith, et al.$^{9}$

\section{Conclusions}

This paper presents and validates the uncoupled TF formulations of the RPTF and RMM methods. This enables development of a simple, but powerful, database analysis tool for the MSFC vibroacoustics team to estimate vibration responses at equipment mounting locations. With this database tool, it will no longer be necessary to use heritage processes that typically require the development of detailed analysis models and/or the collection and processing of substantial ground and flight test data. This puts powerful capability into the hands of the propulsion and vehicle system departments to provide input vibration environment requirements for a new launch vehicle program. In addition to improving the uncoupled formulations with the use of well-known component mode synthesis techniques, future work may involve expanding the methods to accept other types of source environments as input. For instance, the aero-fluctuating pressure environment associated with vehicle ascent is often modeled with what is known as a Corcos model!11 Implementing such a model into the database tool for use with the RPTF method would provide even greater capability for predicting environments across all flight regimes.

\section{Acknowledgments}

The authors would like to acknowledge the fine support provided by the test organization at MSFC. The dedicated and capable staff of the ET40 department has provided outstanding support to complete an earlier set of ambitious acoustic response tests. Additionly, the authors are thankful for the insightful comments and contributions made to this paper by Clay W. Fulcher of MSFC. The financial support of the MSFC Technical Excellence program is also gratefully acknowledged.

\section{References}

\footnotetext{
${ }^{1}$ Kaouk, M., Harrison, P., and Blelloch, P., "Summary of NASA Vibro-Acoustic Technical Interchange Meeting," (unpublished presentation), NASA Kennedy Space Center, Cape Canaveral, FL, Oct. 2009.

${ }^{2}$ Kern, D., "Proposal for Reducing Risk Associated with Vibroacoustic Environments," (unpublished presentation at the NESC Loads and Dynamics Technical Discipline Team annual face to face meeting), Waco, TX, May 2010.

${ }^{3}$ Smith, A., Harrison, P., LaVerde, B., Hunt, R., and Teague, D., "Preliminary Evaluation of Mass Loaded Vehicle Panel Response Estimates and approaches Based on Acoustic Ground Test Results," Proceedings of the Aerospace Testing Seminar, ATS, 2011.

${ }^{4}$ Peck, J., Smith, A., Fulcher, C., LaVerde, B., and Hunt, R., "Development of Component Interface Loads on a Cylindrical
} 
Orthogrid Vehicle Section from Test-Correlated Models of a Curved Panel," Proceedings of the Spacecraft and Launch Vehicle Dynamic Environments Workshop, AIAA, 2011.

${ }^{5}$ Frady, G., Duvall, L., Fulcher, C., LaVerde, B., and Hunt, R., "Test-Anchored Vibration Response Predictions For An Acoustically Energized Curved Orthogrid Panel With Mounted Components," Proceedings of JANNAF's 8th Modeling and Simulation Subcommittee (MSS), JANNAF, 2011.

${ }^{6}$ Majed, A., Henkel, E., and Kolaini, A., "An improved method of structural-borne random vibration mass attenuation," Proceedings of $162^{\text {nd }}$ meeting of the Acoustical Society of America, Acoustical Society of America, 2011.

${ }^{7}$ Maasha, R., Towner, R., LaVerde, B., and Band, J., "Preliminary Correlation Results Summary for Mass Loaded Vehicle Panel Test Article Finite Element Models and Modal Survey Tests," Proceedings of the Spacecraft and Launch Vehicle Dynamic Environments Workshop, AIAA, 2011.

${ }^{8}$ Bendat, J. S. and Piersol, A. G., Random Data: Analysis and Measurement Procedures, Wiley, 1986.

${ }^{9}$ Smith, A. M., Davis, R. B., LaVerde, B. T., Fulcher, C. W., Jones, D. C., Waldon, J. M., and Craigmyle, B. B., "Validation of Measured Damping Trends for Flight-Like Vehicle Panel/Equipment Including a Range of Cable Harness Assemblies," $53^{r d}$ AIAA/ASME/ASCE/AHS/ASC Structures, Structural Dynamics, and Materials Conference, 23-26 April 2012, Honolulu, $H I$, AIAA, 2012.

${ }^{10}$ Craig, R. R. and Bampton, M. C. C., "Coupling of Substructures for Dynamic Analysis," AIAA Journal, Vol. 6, No. 7 , 1968, pp. 1313-1319.

${ }^{11}$ Corcos, G. M., "Resolution of Pressure in Turbulence," Journal of the Acoustical Society of America, Vol. 35, No. 2, 1963, pp. 192-199. 


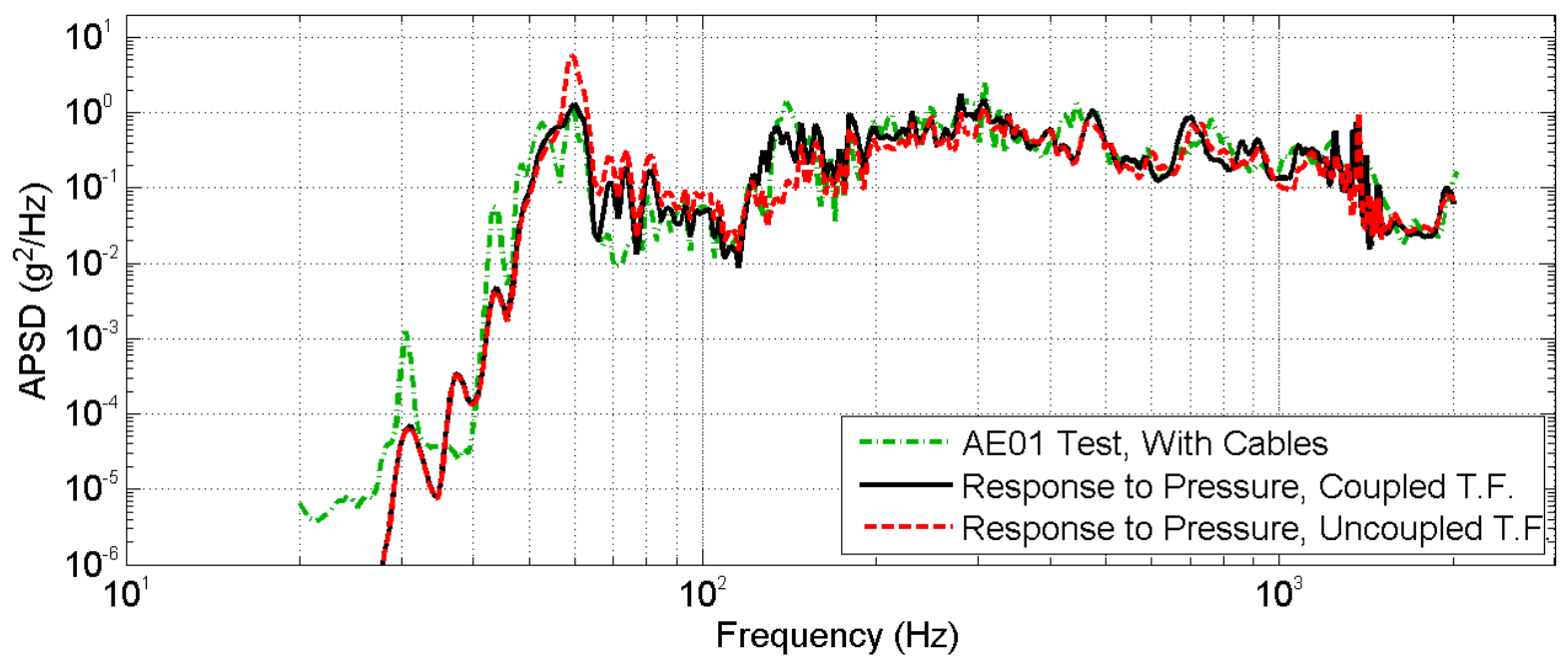

Figure 6. Acceleration response spectra from uncoupled RPTF formulation and the coupled RPTF formulation compared with test data. 